\title{
On Correlating Sonar Images
}

\author{
Richard J. Rikoski, J. Tory Cobb, and Daniel C. Brown \\ Naval Surface Warfare Center Panama City \\ Panama City, Florida 32408 \\ Email: richard.rikoski@navy.mil
}

\begin{abstract}
This paper demonstrates that sonar images correlate badly because target geometry and image fringes correlate in different coordinate systems. It is shown that if the receiver is a line array, all point objects have the same image in a coordinate system with axes of range and the sine of target bearing (the $(r, s)$ coordinate system). Results from an ocean experiment are presented. The ocean experiment shows that after a simple translation, the correlation coefficient between Cartesian images of a radar reflector drop to zero, while the correlation coefficient between images of the same radar reflector in an $(r, s)$ coordinate system hover around 0.9 .
\end{abstract}

\section{INTRODUCTION}

Data association is a fundamental problems in feature-based navigation. When the timescale between observations is small, data association can be considered a tracking problem; when the timescale between observations is large, data association can be considered a recognition problem. One approach to object recognition is to coherently correlate images of targets, an approach that typically fails when applied to sonar images. This paper will show that coherent correlation fails because the point response of a line array is shift invariant only in an $(r, s)$ coordinate system.

Numerous groups have investigated sonar perception. Leonard and Durrant-Whyte [7] developed regions of constant depth (RCDs) to determine correspondence in a sonar scan. Nagatani et al. [8] developed the Arc Transversal Median method to find edges. Mataric and Brooks [2] developed a robot that maintained a fixed distance from walls by tracking without an explicit model. Kleeman and Kuc [5] used the method of images to distinguish corners and walls. Barshan and Kuc [1] developed a sonar that differentiated between convex corners and walls based on amplitude measurements. Kuc [6] developed a sonar that could recognize objects based on waveforms; the sonar adaptively positioned itself to search for unique aspects of the target. Peremans et al. [11] built a tri-aural sonar for estimating the range and bearing to a target. Wijk and Christensen [15], [16] developed Triangulation Based Fusion, a method for mapping vertical edges using a sliding window of recent sonar measurements. Kleeman [4] designed a sonar that used double pulse coding to reject interference and perform classification.

Williams and Mahon [14], in a Great Barrier Reef experiment, combined sonar and vision to do particle filter based localization. In a Baltic Sea experiment, Nygren and Jannson [10] correlated bathymetric sonar images of the sea floor against a prior map for localization. (The images used in this paper differ in that they have an intensity component.)
At the GOATS 2002 experiment in the Ligurian Sea, Rikoski et al. [13] tracked continuously observed objects using a universal feature model and Newman et al. [9] performed SLAM using the tracked features.

\section{Correlating Two Image Patches}

In this paper, correlation will refer to calculating the correlation coefficient between two images or image patches.

Consider an image patch $P_{1}$ from the Cartesian image $I_{1}(x, y)$, centered at $\left(x_{1}, y_{1}\right)$, with width and height $\Delta x$ and $\Delta y$. The statistics of the patch can be calculated by multiplying the original image $I_{1}(x, y)$ against a mask $M(x, y)$, where the mask is defined as being one inside of the patch and zero outside of the patch.

$$
\begin{gathered}
M(x, y)=1 \\
\left|x-x_{1}\right|<\frac{\Delta x}{2},\left|y-y_{1}\right|<\frac{\Delta y}{2} \\
M(x, y)=0 \text { elsewhere. }
\end{gathered}
$$

The variance of $P_{1}$ can be written as

$$
\begin{gathered}
\sigma_{1}^{2}=E\left[P_{1}^{2}\right]-E\left[P_{1}\right]^{2} \\
E\left[P_{1}\right]=\frac{\int_{-\infty}^{\infty} \int_{-\infty}^{\infty} I_{1}(x, y) M(x, y) d x d y}{\int_{-\infty}^{\infty} \int_{-\infty}^{\infty} M(x, y) d x d y} \\
E\left[P_{1}^{2}\right]=\frac{\int_{-\infty}^{\infty} \int_{-\infty}^{\infty} I_{1}^{2}(x, y) M(x, y) d x d y}{\int_{-\infty}^{\infty} \int_{-\infty}^{\infty} M(x, y) d x d y} .
\end{gathered}
$$

In a second image $I_{2}(x, y)$ there is a patch $P_{2}$ that is shifted some amount $(\delta x, \delta y)$ with respect to the first patch. The variance of the second patch as a function of its shift is

$$
\begin{gathered}
\sigma_{2}^{2}(\delta x, \delta y)=E\left[P_{2}(\delta x, \delta y)^{2}\right]-E\left[P_{2}(\delta x, \delta y)\right]^{2} \\
E\left[P_{2}(\delta x, \delta y)\right]=\frac{\int_{-\infty}^{\infty} \int_{-\infty}^{\infty} I_{2}(x+\delta x, y+\delta y) M(x, y) d x d y}{\int_{-\infty}^{\infty} \int_{-\infty}^{\infty} M(x, y) d x d y} \\
E\left[P_{2}^{2}(\delta x, \delta y)\right]=\frac{\int_{-\infty}^{\infty} \int_{-\infty}^{\infty} I_{2}^{2}(x+\delta x, y+\delta y) M(x, y) d x d y}{\int_{-\infty}^{\infty} \int_{-\infty}^{\infty} M(x, y) d x d y}
\end{gathered}
$$

The covariance between the two patches, as a function of the shift, is

$$
\begin{gathered}
\sigma_{12}^{2}(\delta x, \delta y)=E\left[P_{1} P_{2}(\delta x, \delta y)\right]-E\left[P_{1}\right] E\left[P_{2}(\delta x, \delta y)\right] \\
E\left[P_{1} P_{2}(\delta x, \delta y)\right]=
\end{gathered}
$$




$$
\frac{\int_{-\infty}^{\infty} \int_{-\infty}^{\infty} I_{1}(x, y) M(x, y) I_{2}(x+\delta x, y+\delta y) d x d y}{\int_{-\infty}^{\infty} \int_{-\infty}^{\infty} M(x, y) d x d y}
$$

The correlation coefficient between the two patches is then

$$
\rho(\delta x, \delta y)=\frac{\sigma_{12}(\delta x, \delta y)}{\sqrt{\sigma_{1} \sigma_{2}(\delta x, \delta y)}}
$$

The correlation coefficient for all possible shifts can be efficiently calculated in the frequency domain.

\section{Correlating Images of Point Objects}

Without rigorous proof, assume that the correlation coefficient $\rho(\delta x, \delta y)$ equals unity when two image patches are identical except for a shift of $(\delta x, \delta y)$, and is not equal to unity when the images differ in some way. Therefore, it would be desirable to correlate images of a target in a coordinate system where the image is invariant to shifts. It will now be shown that the image of a point object created by a line array in a two dimensional environment is position dependent in Cartesian and polar coordinates, but not in $(r, s)$ coordinates.

\section{A. Imaging a Point in Cartesian Coordinates}

Assume a sonar with an omnidirectional transmitter at $(0,0)$ that transmits a signal $S(t)$ and has $N$ omnidirectional receiving elements along the $y$ axis, the $i$ th being at position $\left(0, y_{i}\right)$ and receiving signal $S_{i}(t)$. A point target at position $\left(x_{p}, y_{p}\right)$ is imaged using the signals $S_{i}(t), i \in\{1, \ldots, N\}$.

Given the system geometry, the distance $d_{t}$ from the transmitter to the point object is

$$
d_{t}=\sqrt{x_{p}^{2}+y_{p}^{2}} .
$$

The distance $d_{i}$ from each receiving element to the point target

$$
d_{i}=\sqrt{x_{p}^{2}+\left(y_{p}-y_{i}\right)^{2}} .
$$

Therefore, the distance-of-flight $D O F_{i}$ the sound travels from the transmitter to each receiving element is

$$
D O F_{i}=d_{t}+d_{i}=\sqrt{x_{p}^{2}+y_{p}^{2}}+\sqrt{x_{p}^{2}+\left(y_{p}-y_{i}\right)^{2}} .
$$

Assuming the point is far from the array $\left(r>\frac{L^{2}}{\lambda}\right.$, where $\mathrm{L}$ is the length of the array and $\lambda$ is the representative wavelength), this can be rewritten as

$$
D O F_{i} \approx 2 \sqrt{x_{p}^{2}+y_{p}^{2}}-y_{i} \sin \left(\theta_{p}\right)
$$

where $\theta_{p}$ is defined as

$$
\theta_{p}=\arctan \frac{y_{p}}{x_{p}} .
$$

Denoting $c$ as the speed of sound, the time-of-flight $T O F_{i}$ of the signal for each element is

$$
T O F_{i}=\frac{D O F_{i}}{c} \approx \frac{2 \sqrt{x_{p}^{2}+y_{p}^{2}}-y_{i} \sin \left(\theta_{p}\right)}{c} .
$$

Assuming the reflection from the point object is simply a delayed version of the transmitted signal $S(t)$, the received signal can be rewritten as

$$
S_{i}(t)=S\left(t-T O F_{i}\right) \approx S\left(t-\frac{2 \sqrt{x_{p}^{2}+y_{p}^{2}}-y_{i} \sin \left(\theta_{p}\right)}{c}\right) .
$$

Now, consider an image $I(x, y)$. The image value corresponding to location $(x, y)$ will be defined as

$$
I(x, y)=\sum_{i=1}^{N} S_{i}\left(\operatorname{TOF}_{i}(x, y)\right)
$$

where $\operatorname{TOF}_{i}(x, y)$ is the time of flight from the transmitter to position $(x, y)$ to receiving element $i$

$$
\begin{aligned}
\operatorname{TOF}_{i}(x, y) & =\frac{\sqrt{x^{2}+y^{2}}+\sqrt{x^{2}+\left(y-y_{i}\right)^{2}}}{c} \\
& \approx \frac{2 \sqrt{x^{2}+y^{2}}-y_{i} \sin \theta}{c}
\end{aligned}
$$

where $\theta$ is defined as

$$
\theta=\arctan \frac{y}{x} .
$$

The equation for the image can either be rewritten in terms of the element signals or delayed versions of the transmitted signal

$$
\begin{gathered}
I(x, y) \approx \sum_{i=1}^{N} S_{i}\left(\frac{2 \sqrt{x^{2}+y^{2}}-y_{i} \sin \theta}{c}\right) \\
=\sum_{i=1}^{N} S\left(\frac{2 \sqrt{x^{2}+y^{2}}-y_{i} \sin \theta}{c}-\frac{2 \sqrt{x_{p}^{2}+y_{p}^{2}}-y_{i} \sin \left(\theta_{p}\right)}{c}\right)
\end{gathered}
$$

It is worth noting that at the position in the image corresponding to the location of the point $\left(x_{p}, y_{p}\right)$, the image value is

$$
I\left(x_{p}, y_{p}\right)=\sum_{i=1}^{N} S(0)
$$

which is independent of the target location. The value at $\left(x_{p}, y_{p}\right)$ is shift invariant. Now, consider the value at a location $\left(x_{p}+\Delta x, y_{p}+\Delta y\right)$ near the point. At this new position, the image value is

$$
\begin{gathered}
I\left(x_{p}+\Delta x, y_{p}+\Delta y\right) \approx \\
\sum_{i=1}^{N} S\left(\frac{2 \sqrt{\left(x_{p}+\Delta x\right)^{2}+\left(y_{p}+\Delta y\right)^{2}}-y_{i} \sin \theta}{c}\right. \\
\left.-\frac{2 \sqrt{x_{p}^{2}+y_{p}^{2}}-y_{i} \sin \theta_{p}}{c}\right)
\end{gathered}
$$

which is a function of $\left(x_{p}, y_{p}\right)$. In other words, if the target were at a different location in the image $\left(x_{p}^{\prime}, y_{p}^{\prime}\right)$, the image value $(\Delta x, \Delta y)$ away from the target position would be different than it would be if the target were at $\left(x_{p}, y_{p}\right)$.

The Cartesian image of a point target is dependent on where it is with respect to a line array. Not only will two noiseless 

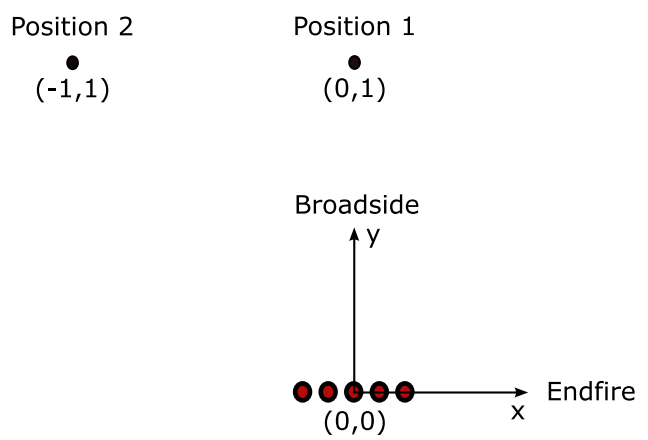

Line Array

Fig. 1. Diagram of simulated setup. The point target was translated from Position 1 to Position 2.

images fail to yield a correlation coefficient of one, but the peak value can correspond to a highly biased shift.

To illustrate this, consider the simulation shown in Figures 1- 4. (A simulation is used because Cartesian correlations break down so dramatically that it is difficult to explain in an environment of any complexity.) To make better use of the aspect ratio of the figures, the array is placed on the $x$ axis, centered at $(0,0)$. The transmitted signal is a $100 \mathrm{kHz}$ 5 cycle tone. A point object is imaged at two positions, labeled Positions 1 and 2. The resulting images are shown in Figure 2. In Figures 3(a) and 3(b), we see the zoomed out versions of the images from Figure 2 replotted in decibels. The correlation coefficient image is shown in Figure 4(a). Clearly, although the actual target shift is $(-1,0)$, the peak correlation coefficient corresponds to a shift of $(0,0.414)$. Looking at Figure 4(b), it is obvious why the incorrect shift yields the peak. At the peak correlation coefficient, the image fringes are parallel, at the true shift, they are misaligned. The fringes do not correlate best where the target geometry correlates best.

\section{B. Imaging a Point in Polar Coordinates}

If Eq. 16 is rewritten in polar coordinates, the pixel position becomes $(r, \theta)$, and the target position becomes $\left(r_{p}, \theta_{p}\right)$. The image has pixels of size $(d r, d \theta)$. The image equation becomes

$$
\begin{gathered}
I(r, \theta) \approx \sum_{i=1}^{N} S_{i}\left(\frac{2 r-y_{i} \sin \theta}{c}\right) \\
=\sum_{i=1}^{N} S\left(\frac{2 r-y_{i} \sin \theta}{c}-\frac{2 r_{p}-y_{i} \sin \left(\theta_{p}\right)}{c}\right) .
\end{gathered}
$$

The value at a point $\left(r_{p}+\Delta r, \theta_{p}+\Delta \theta\right)$ is

$$
\begin{gathered}
I\left(r_{p}+\Delta r, \theta_{p}+\Delta \theta\right) \approx \sum_{i=1}^{N} S_{i}\left(\frac{2\left(r_{p}+\Delta r\right)-y_{i} \sin \left(\theta_{p}+\Delta \theta\right)}{c}\right) \\
=\sum_{i=1}^{N} S\left(\frac{2 \Delta r-y_{i} \sin \left(\theta_{p}+\Delta \theta\right)+y_{i} \sin \left(\theta_{p}\right)}{c}\right) .
\end{gathered}
$$

In this case, the image is independent of the radial coordinate $r_{p}$, but it is still a function of the azimuthal coordinate.
Essentially, this is because the angular resolution of the array is a function of angle; the array has better resolution at broadside than at endfire. As the target moves from broadside to endfire, its image is dilated in the $\theta$ direction.

\section{Imaging a Point in $(r, s)$ Coordinates}

Finally, if Eq. 16 is rewritten in $(r, s)$ coordinates, where $r$ is range and $s=\sin \theta$, it becomes

$$
\begin{gathered}
I(r, s) \approx \sum_{i=1}^{N} S_{i}\left(\frac{2 r-y_{i} s}{c}\right) \\
=\sum_{i=1}^{N} S\left(\frac{2 r-y_{i} s}{c}-\frac{\left.2 r_{p}-y_{i} s_{p}\right)}{c}\right) .
\end{gathered}
$$

The image value at a point $\left(r_{p}+\Delta r, s_{p}+\Delta s\right)$ is

$$
\begin{gathered}
I\left(r_{p}+\Delta r, s_{p}+\Delta s\right) \approx \sum_{i=1}^{N} S_{i}\left(\frac{2\left(r_{p}+\Delta r\right)-y_{i}\left(s_{p}+\Delta s\right)}{c}\right) \\
=\sum_{i=1}^{N} S\left(\frac{\left.2 \Delta r-y_{i} \Delta s\right)}{c}\right) .
\end{gathered}
$$

Regardless of the target position $\left(r_{p}, s_{p}\right)$, the value at $\left(r_{p}+\right.$ $\left.\Delta r, s_{p}+\Delta s\right)$ is the same. Since the image is shift invariant, it perfectly correlates with itself in the noiseless case.

Having established that images of point objects are shift invariant in the $(r, s)$ coordinate system, it is obvious why sonar images correlate poorly. A complex target can be thought of as a collection of point scatterers, and the geometry of the point scatterers is not shift invariant in the $(r, s)$ coordinate system. Image fringes correlate in $(r, s)$, target geometry correlates in $(x, y)$, and the image of a geometrically extensive target with significant fringing correlates in neither. Definitions of "extensive geometry" and "significant fringing" will be left for a future paper. The question then becomes how well targets can be correlated over small perturbations, and what the limits are on target size. This is left as future work.

\section{EXPERIMENTAL RESULTS}

Two experiments will be presented in this section. The first experiment demonstrates correlating images in the $(r, s)$ coordinate system using a simple sonar in a testing tank. The second experiment demonstrates correlating sonar images made by a moving robot in the ocean.

\section{A. Tank Experiment}

To demonstrate correlating $(r, s)$ images, a simple sonar was constructed for a test tank. A $100 \mathrm{kHz}$ transducer with a $5 \mathrm{~cm}$ diameter circular aperture was used as a transmitter. Five $125 \mathrm{kHz}$ broadband spherical hydrophones, spaced $2.5 \mathrm{~cm}$ apart, were used as a line array. The transmitter was separated slightly (less than $10 \mathrm{~cm}$ ) from the receiver and remained fixed throughout the experiment. To vary the target bearing, the receiver was mounted on a rotatable rod. The target was placed 1.25 meters in front of the transmitter. 


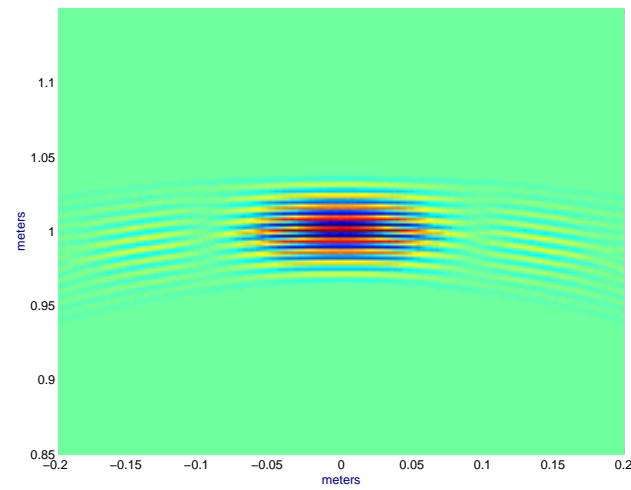

(a) Simulated image of a point target at Position 1.

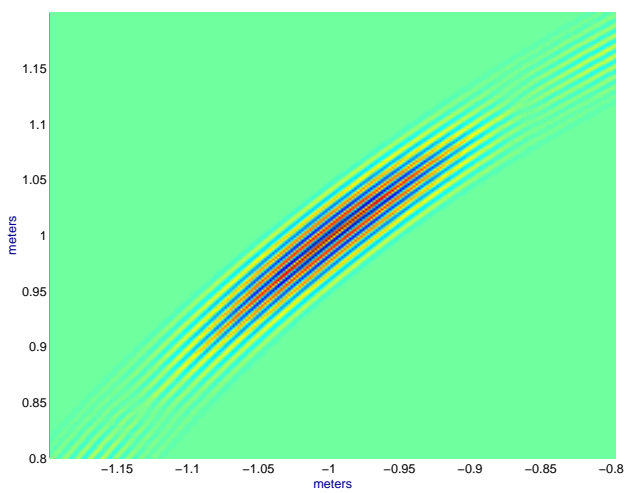

(b) Simulated image of a point target at Position 2.

Fig. 2. Simulated images of a point target made by a line array using the target locations in Figure 1. Clearly, the fringes in the two images have different orientations.

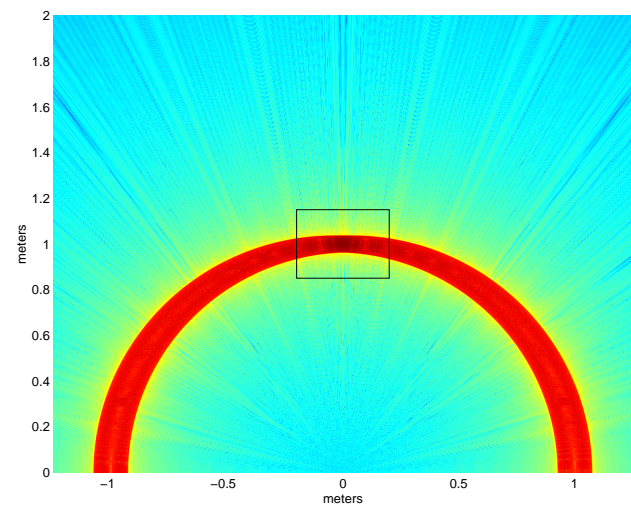

(a) Zoomed out image from Position 1 in $\mathrm{dB}$.

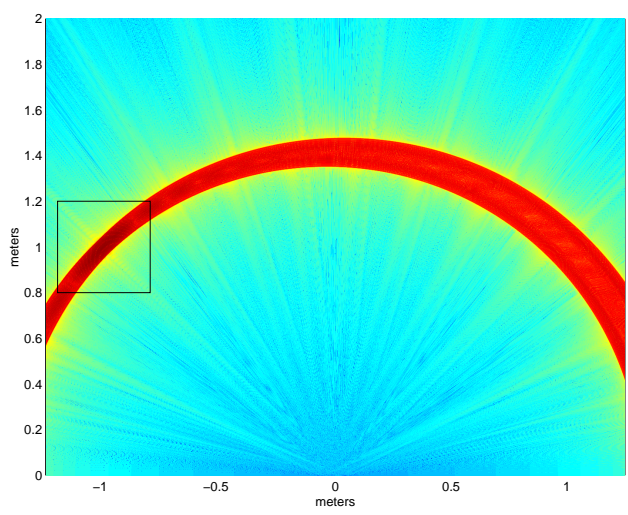

(b) Zoomed out image from Position 2 in $\mathrm{dB}$.

Fig. 3. By expanding the simulated images and plotting them in decibels, it is evident that there exist low energy portions of the two images that are parallel. These are the portions of the image that correlate best. The boxed portions of the images are what are shown in Figure 2. The radial streaking is due to Gibbs phenomena in the simulator, which is only observable when plotted in decibels.

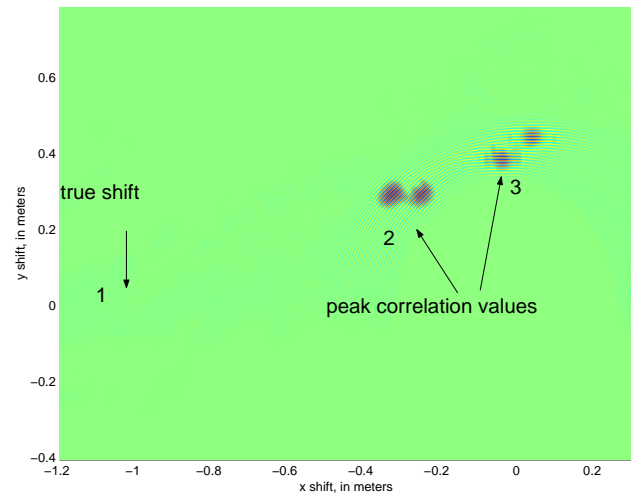

(a) The correlation of the simulated images.

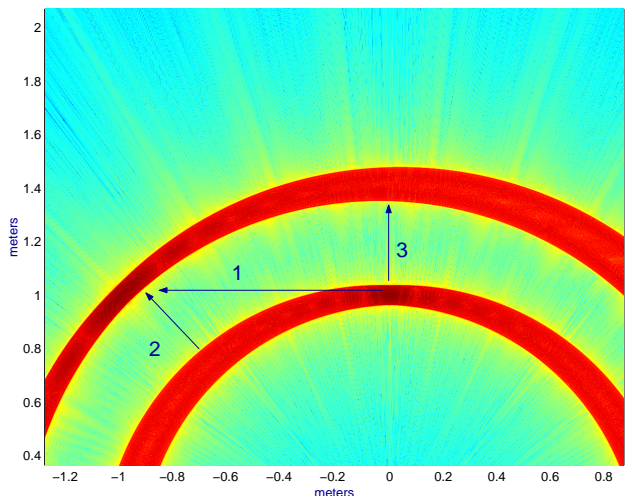

(b) The cause of the biased peaks in the correlation of the simulated images.

Fig. 4. From Figure 1, it is evident that the target shifted 1 meter to the left between images. This shift is labeled as 1 in the images, and yields a very low correlation coefficient. The peaks in the correlation image, labeled as 2 and 3 , correspond to shifting the peak values in the images over to parallel fringes. This leads to highly biased peak correlations. 


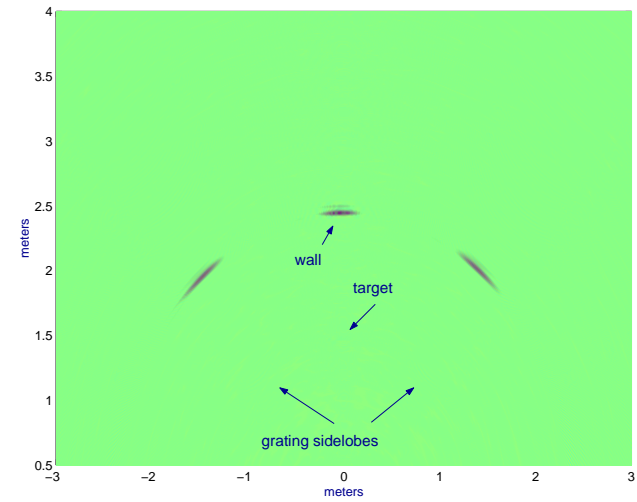

(a) Cartesian image of the tank environment.

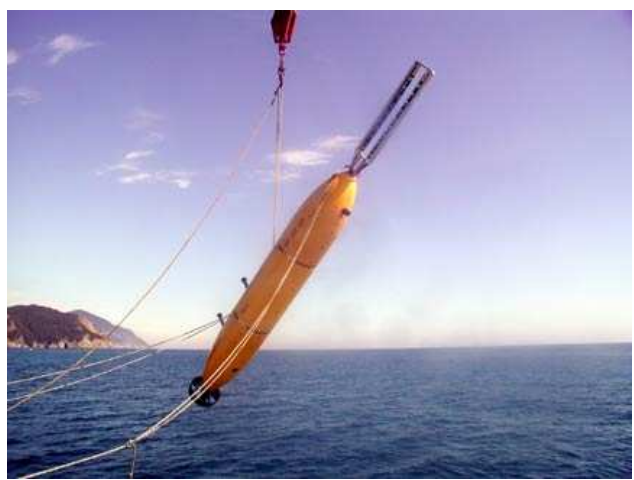

(b) Robot used in the ocean experiment.

Fig. 5. For the tank experiment, the target was placed in the middle of a testing tank, about a meter from a wall. The robot on the right was used for the ocean experiment.

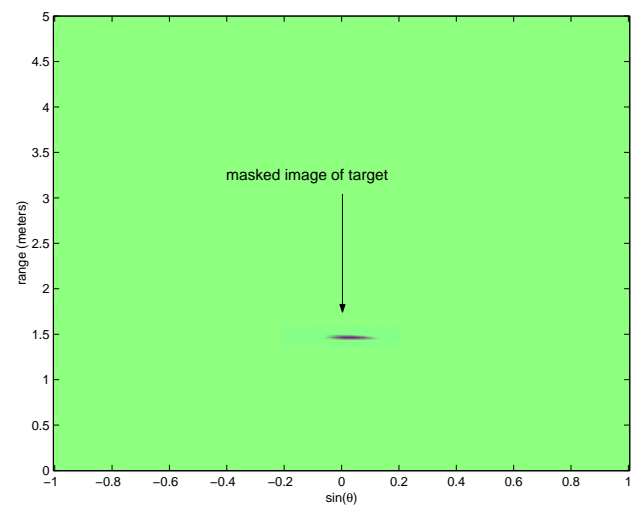

(a) The masked broadside $(r, s)$ image of an oil filled sphere.

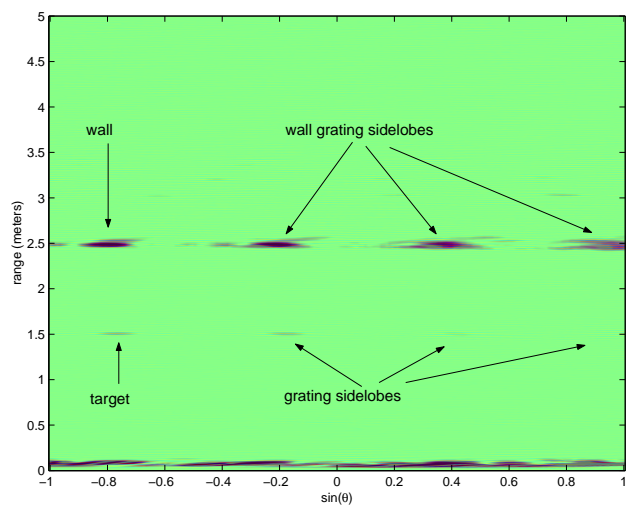

(b) The $(r, s)$ image of an oil filled sphere at a $60^{\circ}$ angle to the array.

Fig. 6. Oil filled sphere images.

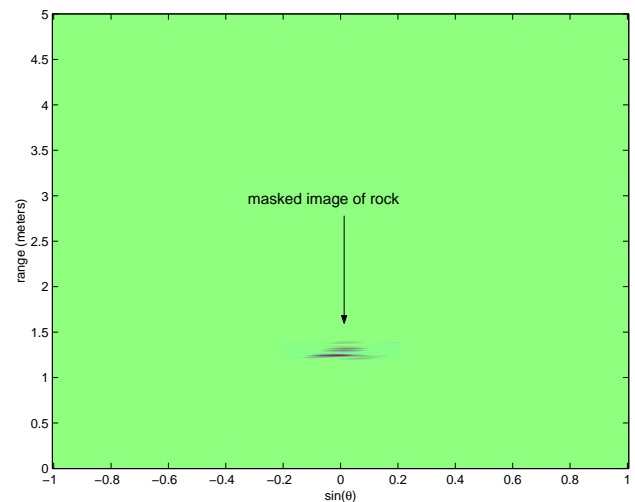

(a) The broadside $(r, s)$ image of a rock.

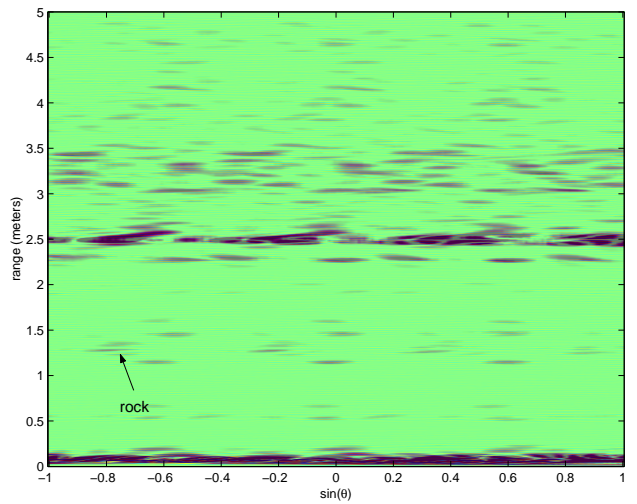

(b) The $(r, s)$ image of a rock at a $60^{\circ}$ angle to the array.

Fig. 7. Images of a rock suspended in a testing tank. 


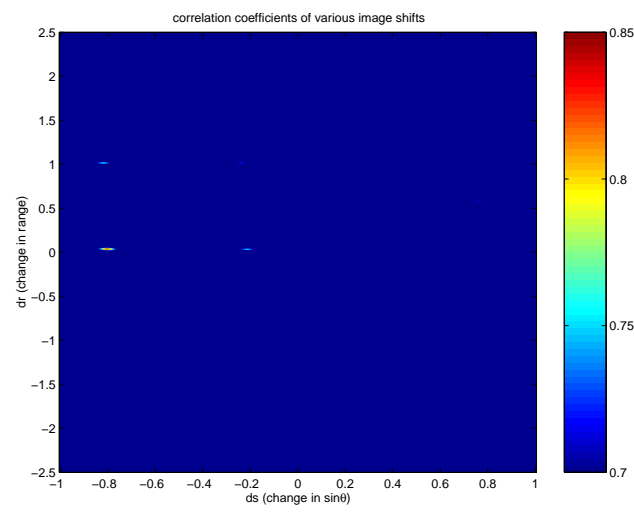

(a) The correlation image for the oil filled sphere.

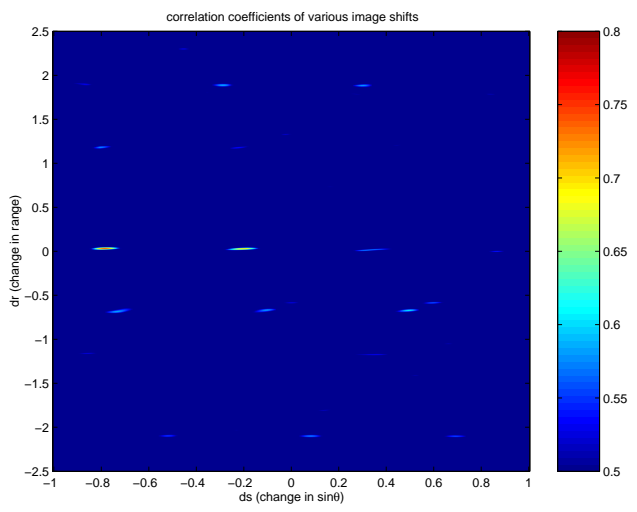

(b) The correlation image for the rock.

Fig. 8. The correlation images of the two targets. In both cases, the peaks correspond to a shift of approximately $60^{\circ}$ in the target location. Due to the crude experimental apparatus (an array on a stick) it is impossible to make any strong statements about the errors in the peak values, but clearly the biases seen in the Cartesian case are not evident. The peak correlation coefficient for the sphere is .71, the peak correlation coefficient for the rock is .74.

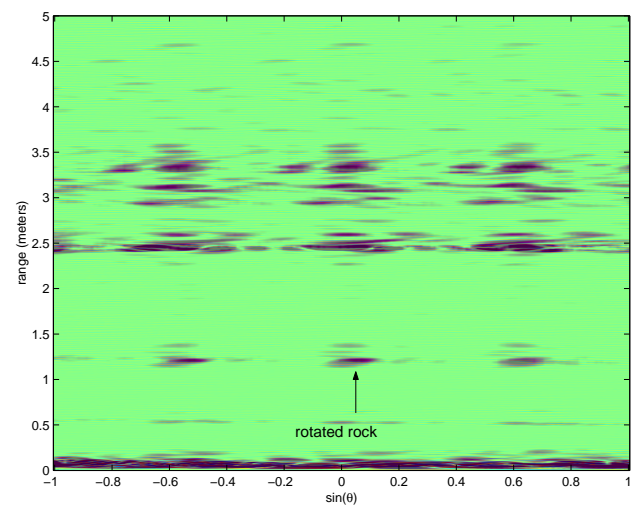

(a) The $(r, s)$ image of a rock at broadside to the array after being rotated approximately $60^{\circ}$.

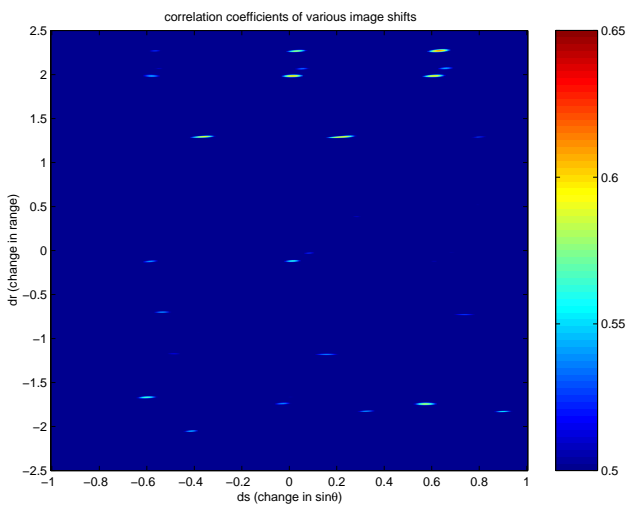

(b) The correlation for the rotated rock. The true shift is weakly shown at $(0,0)$, higher correlation coefficients occur for incorrect shifts.

Fig. 9. The image and correlation after rotating the rock $60^{\circ}$ while keeping it at broadside to the array. Correlating the rock template with the rotated rock yields a correlation coefficient .55, other objects in the environment yield higher correlation coefficients.

In the first tank experiment, an oil filled sphere was used as a target. Images from broadside and $60^{\circ}$ were correlated. In the second experiment, images of a rock at broadside and at $60^{\circ}$ were correlated. In the last experiment, the rock was placed at broadside and rotated $60^{\circ}$ between images. In the first two experiments (Figures 6 and 7), the peak correlations corresponded to the correct shift. In the third experiment, the peak correlation did not correspond to the correct shift. This is because the target was aspect dependent.

\section{B. GOATS Experiment}

At NATO SACLANT's GOATS 2002 experiment off the coast of Framura, Italy, the Bluefin Robotics Odyssey III class autonomous underwater vehicle Caribou used a 16-element dual line array $5-25 \mathrm{kHz}$ broadside synthetic aperture sonar to gather acoustic data in a target field. The results presented here are from the data set referred to as SAS_15_6_2002_-_16_11, which was also featured in [9], [12], [13]. In this data set, the robot circled a target field of radar reflectors.
A typical sonar image of a radar reflector is shown in Figure 10(a). This image was generated using one of the line arrays on Caribou. Since a radar reflector is designed to act as a strong point object, all images of radar reflectors were essentially the same. The only object in the data that looked different was the image of the sea floor, which is shown in Figure 10(b). Although the image of the sea floor is similar to the image of the radar reflector (due to a strong specular component), it is broader and longer due to diffuse scattering.

In [5], point and planar objects were differentiated by the difference in arrivals between elements. Because planar objects acted as mirrors, causing the receiver to see an image of the transmitter, by looking at the signal arrival times across the elements points and planes could be differentiated. This is a very powerful technique. However, it only works if the target is in the nearfield of the array, a condition that does not hold in this case. Therefore, the difference in the angular extent of the radar reflector and bottom image is not due to this phenomena.

The radar reflector and sea floor were tracked using the 
technique described in [13]. Time series of the range to the two are shown in Figure 11. Range and bearing were constructed from images of the target, images which were also correlated.

To show the correlation between images, a template image was chosen for each target. The image from timestep 2930 was chosen for the radar reflector; the image from 1244 was chosen for the sea floor. Those images are shown in Figure 10. The image template was correlated against all other images in the time series of observations. The correlation of the image against itself is not graphed since its coefficient is one.

Figure 12(a) shows the time series of correlation coefficients for the radar reflector. At the top (in black) are the peak correlation coefficients for each timestep derived by comparing $(r, s)$ images to the template. The target is $50 \mathrm{~m}$ away, and the correlation coefficients are approximately 0.9 . The correlations drop as the sonar beam moves past the target.

In the middle (in green) are the correlation coefficients for the $(r, s)$ radar reflector images and the template of the sea floor. The two correlate fairly well, although not as well as correlating the radar reflector with itself. This is because of the strong specular reflection in the bottom bounce.

On the bottom (in black) are the correlation coefficients for the Cartesian images of the radar reflector and the Cartesian radar template. Some may find it surprising that the coefficient reaches zero, as zero correlation is quite rare. A zero occurs because the covariance between images is zero. The covariance is obtained by taking the mean value of the product of two images. Looking at the images in Figure 2, it is clear that multiplying the two together will lead to an "egg carton" pattern. Since the mean of that pattern is zero, the covariance is zero, and the expected correlation coefficient is zero.

\section{CONCLUSION}

This paper has shown that line array images of point targets correlate well in an $(r, s)$ coordinate system. In the noiseless case, the correlation coefficient between any two images of a point target will always be one. For Cartesian coordinates, the correlation coefficient falls off quickly as the difference between target bearings increase. This is because the fringe pattern rotates to focus on the receiver. This leads to a fundamental problem. A complex target can be thought of as a collection of point scatterers. If those point scatterers are translated, their positions will correlate perfectly in a Cartesian coordinate system, but their sonar images will quickly decorrelate in a Cartesian coordinate system as this individual fringe patterns rotate and dialate. In an $(r, s)$ coordinate system, as the collection of point scatterers their fringes will correlate but the configuration of the constellation will be transformed; the geometry will not correlate. Correlating images of targets over a wide range of aspects is unlikely to be successful in the general case.

Having shown that sonar image correlation works for simple targets but fails for complex targets, one naturally wonders about the broader impacts on sonar perception. What does this imply how a robot ought to perceive its environment using sonar? Is correlation ever an acceptable technique? How should a robot recognize objects from different vantage points?

Our belief is that correlation will be shown to be acceptable within limited regimes, that there will be a tradeoff between object complexity and acceptable translations, and that large complex objects will decorrelate faster than small or simple objects. In a SLAM context, images of near objects will decorrelate faster than images of distant objects for a given translation, as the change in angle will be smaller. Landmarks should be distinct and distant.

\section{ACKNOWLEDGMENT}

The authors would like to thank John Leonard and Joe Lopes for providing the sonar equipment used in the first experiment. They would also like to thank Henrik Schmidt, John Leonard, Paul Newman, Rob Damus, Jim Morash, Sam Desset, Don Eickstedt, Joe Edwards, Chryssostomos Chryssostomidis, Matt Grund, and the crew of the Alliance, for their help with the GOATS cruise.

\section{REFERENCES}

[1] B. Barshan and R. Kuc, Differentiating Sonar Reflections from Corners and Planes by Employing an Intelligent Sensor, IEEE Transactions on Pattern Analysis and Machine Intelligence, Vol. 12, No. 6, 1990.

[2] R. Brooks, Cambrian Intelligence: The Early History of the New AI, The MIT Press, 1999.

[3] I. Dyer, Fundamentals of Underwater Sound Applications, Course notes for MIT Dept. of Ocean Engineering class 13.851, 1993.

[4] L. Kleeman, Advanced Sonar with Velocity Compensation, International Journal of Robotics Research, Vol. 23, No. 2, 2004.

[5] L. Kleeman and R. Kuc, Mobile Robot Sonar for Target Localization and Classification, International Journal of Robotics Research, Vol. 14, No. 4, 1995.

[6] R. Kuc, Fusing Binaural Sonar Information for Object Recognition, IEEE/SICE/RSJ International Conference on Multisensor Fusion and Integration for Intelligent Systems, 1996.

[7] J. Leonard, Directed Sonar Sensing for Mobile Robot Navigation, $\mathrm{PhD}$ thesis, Oxford University, 1990.

[8] K. Nagatani, H. Choset, and N. Lazar, The Arc-Transversal Median Algorithm: an Approach to Increasing Ultrasonic Sensor Accuracy, IEEE International Conference on Robotics and Automation, 1999.

[9] P. Newman, J. Leonard, and R. Rikoski, Towards Constant-Time SLAM on an Autonomous Underwater Vehicle Using Synthetic Aperture Sonar, International Symposium on Robotics Research, 2003.

[10] I. Nygren and M. Jansson, Terrain Navigation for Underwater Vehicles Using the Correlator Method, IEEE Journal of Oceanic Engineering, Vol. 29, No. 3, 2004

[11] H. Peremans, K. Audnaert, and C. Van, A high-resolution sensor based on tri-aural perception, IEEE Transactions on Robotics And Automation, Vol. 9, No. 1, 1993.

[12] R. Rikoski, Dynamic Sonar Perception, PhD thesis, MIT, 2003.

[13] R. Rikoski, J. Leonard, P. Newman, and H. Schmidt, Trajectory Sonar Perception in the Ligurian Sea, International Symposium on Experimental Robotics, 2004.

[14] S. Williams and I. Mahon, Terrain Aided Localisation and Mapping for Marine Environments, International Symposium on Experimental Robotics, 2004.

[15] O. Wijk, Triangulation Based Fusion of Sonar Data with Application in Mobile Robot Mapping and Localization, Robotics and Autonomous Systems, PhD thesis, Royal Institute of Technology, 2001.

[16] O. Wijk and H. Christensen, Triangulation Based Fusion of Sonar Data with Application in Robot Pose Tracking, IEEE Transactions on Robotics And Automation, Vol. 16, No. 6, 2000. 


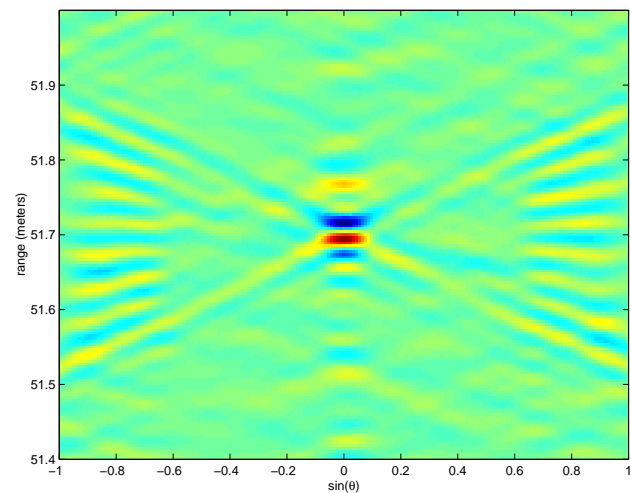

(a) An $(r, s)$ image of a radar reflector.

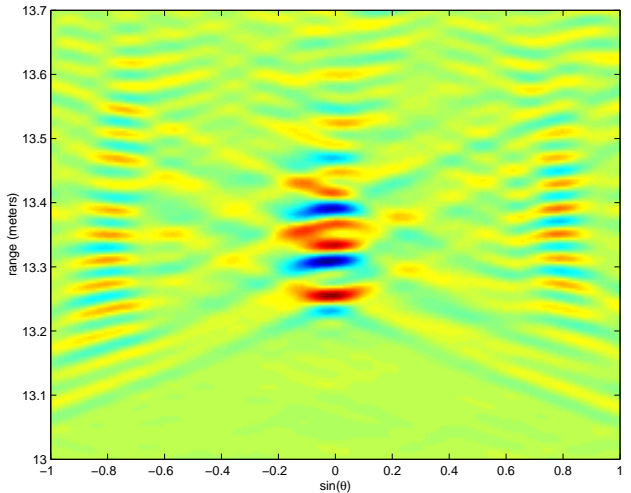

(b) An $(r, s)$ image of the sea floor.

Fig. 10. Typical images of a radar reflector and the bottom. The central portions of these images were used as the templates for the correlations shown in Figure 12.

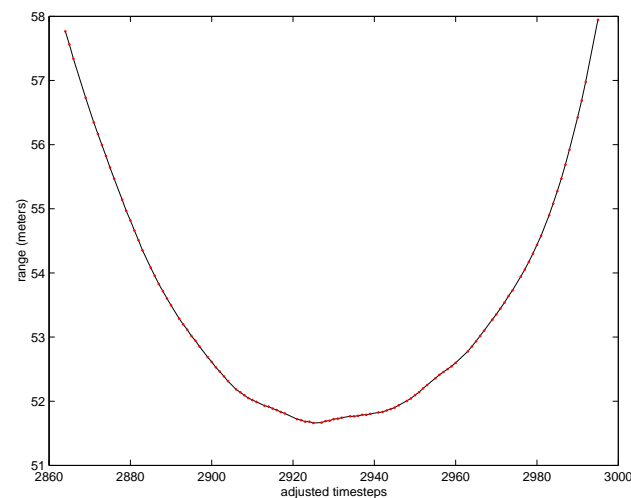

(a) The time series of ranges measurements of the radar reflector. This is the target that was tracked in Figure 6 of [13].

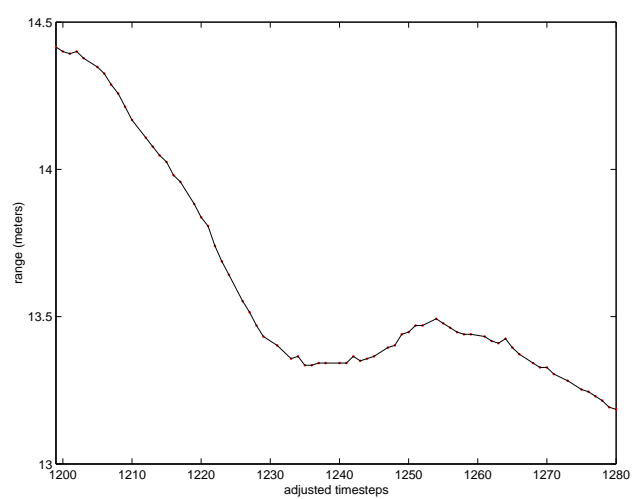

(b) The time series of range measurements of the sea floor used in Figure 12(b).

Fig. 11. Time series of measurements.

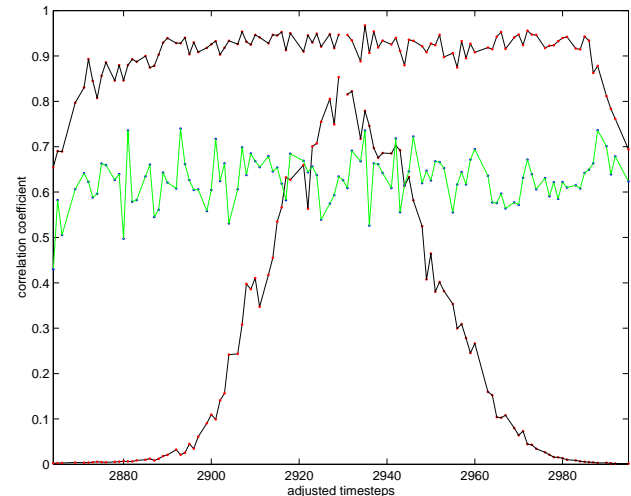

(a) Correlation coefficients of images of a radar reflector. The top line shows the correlation coefficients when the $(r, s)$ image from timestep 2930 is correlated against the other images. The middle line (in green) shows the correlation coefficients when the radar reflector is correlated against the seafloor template. The bottom line shows the correlation between cartesian images of the point target, using the cartesian image from timestep 2930 again as the template.

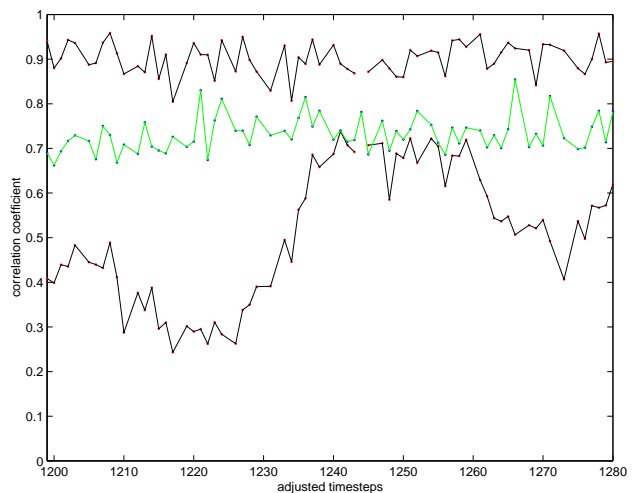

(b) Correlation coefficients of images of the bottom. The top line shows the correlation between $(r, s)$ images of the sea floor and the $(r, s)$ image from timestep 1244. The middle line shows the correlation between $(r, s)$ images of the sea floor and the radar reflector template. The bottom line show the correlation between Cartesian images of the sea floor.

Fig. 12. Time series of correlation coefficients for objects tracked in the ocean. 